Pfleger, Florian; Rapp, Christoph; Manhart, Michael Analysis of the Temporal Evolution of the Sediment Movement in the Vicinity of a Cylindrical Bridge Pier

Verfügbar unter / Available at:

https://hdl.handle.net/20.500.11970/100285

Vorgeschlagene Zitierweise / Suggested citation:

Pfleger, Florian; Rapp, Christoph; Manhart, Michael (2010): Analysis of the Temporal Evolution of the Sediment Movement in the Vicinity of a Cylindrical Bridge Pier. In: Burns, Susan E.; Bhatia, Shobha K.; Avila, Catherine M. C.; Hunt, Beatrice E. (Hg.): Proceedings 5th International Conference on Scour and Erosion (ICSE-5), November 7-10, 2010, San Francisco, USA. Reston, Va.: American Society of Civil Engineers. S. 658-667. 


\title{
Analysis of the Temporal Evolution of the Sediment Movement in the Vicinity of a Cylindrical Bridge Pier
}

\author{
Florian Pfleger ${ }^{1}$, Christoph Rapp ${ }^{2}$, Michael Manhart ${ }^{3}$
}

1 Dipl.-Ing., Fachgebiet Hydromechanik, Technische Universität München, Arcisstr. 21,80333 München, Germany, f.pfleger@bv.tum.de, +49/89/28922419

2 Dr.-Ing., Fachgebiet Hydromechanik, Technische Universität München, Arcisstr.

21, 80333 München, Germany, ch.rapp@bv.tum.de

3 Prof. Dr.-Ing. habil., Fachgebiet Hydromechanik, Technische Universität München, Arcisstr. 21, 80333 München, Germany, m.manhart@bv.tum.de

\begin{abstract}
A detailed analysis of the sediment movement in the vicinity of a cylindrical pier is presented. The experiments were conducted in a laboratory flume. The movement of the sediment grains was detected and evaluated by image processing techniques at several stages of the scouring process. The pictures of the sediment surface were captured with a CMOS-camera from above through a plexiglass plate which was slightly submerged on the water surface. $20 \%$ of the sediment grains were colored black to assure a significant contrast. The experiments were conducted for $20 \mathrm{~h}$ at $80 \%$ of the critical section averaged velocity. The results of the study comprise time-averaged moving directions and magnitudes, scatterplots of the displacement events and transport intensities at a high spatial resolution for all scouring stages. The temporal evolution of the main scouring agents (tangential velocity components and the horseshoe-vortex system) and their importance during the particular stages were evaluated. The development of the grain velocity magnitudes, the dispersion of movement events and the transport intensities are discussed.
\end{abstract}

\section{INTRODUCTION}

Despite a notable amount of scientific investigations on scouring around cylindrical piers the prediction of the maximum scour depth and the temporal evolution of the scour hole is still not possible with a satisfying reliability. Most of the scour formula available are based on experimental laboratory investigations of the scour depth evolution. Due to the specific conditions of these different experiments their field of application is limited. This fact leads to significant deviations of the predicted scour depth and temporal evolution in comparative calculations (Link (2006)). For the development of a more universal approach for the prediction of scour at bridge constructions further detailed investigations of the flow field and the related erosion processes are necessary. Additionally a great demand arises for these experimental studies to improve and validate numerical models for the simulation of scouring processes.

Many investigations on the main influencing factors of the scour hole development (flow field, erosion processes, scour hole geometry) can be found in the literature. Hjorth (1975) studied the flow field and the bed shear stress at the initial stage (flat bed), Melville (1975) and Melville and Raudkivi (1977) measured velocities near the bed and in radial planes and described the erosion processes for initial, intermediate 
and equilibrium scour holes. Zanke (1982) also gave a detailed description of the erosion processes and their evolution in time. Dargahi (1990) analysed the horseshoe vortex system (HV-system) at the pier front and related his findings to the development of the scour hole geometry. Dey and Raikar (2007) measured the flow field in radial planes with an ADV-probe for several evolution stages respectively and analysed the HV-system. Unger and Hager (2007) conducted PIV measurements in several vertical and horizontal planes around a half-cylinder mounted at the side wall of a flume and analysed the HV characteristics.

During the last years several researchers designed image processing based methods for the measurement of sediment movement in laboratory flumes. Pilotti et al. (1997), Sechet and LeGuennec (1999), Keshavarzy and Ball (1999) and Papanicolaou et al. (1999) recorded image series of particles moving along the flume bottom in uniform open channel flow and processed them manually or automatically. Radice et al. (2008) applied image processing techniques to map the sediment movement in the vicinity of abutments.

In this work the kinematics of the sediment erosion processes in the vicinity of a cylindrical bridge pier are analysed. The grain movements were mapped in image series and analysed via digital image processing techniques. Eight different stages of the scour evolution (after 5,30,60,120,240,480,720 and $1200 \mathrm{~min}$ ) were analysed and the qualitative results of the above mentioned literature were compared to these findings.

\section{EXPERIMENTAL SETUP}

In the following the experimental setup is presented. A more detailed description can be found in Pfleger et al. (2010).

\section{Flume}

The experiments were conducted in the Hydromechanics Laboratory of the Technische Universität München. $16 \mathrm{~m}$ downstream of the inlet of a $1.20 \mathrm{~m}$ wide flume a cylindrical pier $(D=0.10 \mathrm{~m})$ was embedded in the center of the flume in a $0.30 \mathrm{~m}$ deep sand bed. $6.5 \mathrm{~m}$ upstream of the cylinder the bottom was elevated and covered with a sediment layer. Two flow straighteners of different mesh size were placed in the approach flow.

\section{Sediment}

Uniform natural coarse sand with a representative diameter $d_{50}=1.9 \mathrm{~mm}$ was used. The angle of repose was measured to be $30^{\circ}$. $20 \%$ of the grains were colored black to ensure a significant contrast for the image processing.

\section{Hydraulic conditions}

The presented experiment was conducted at a flow depth of $0.15 \mathrm{~m}$ and a section averaged flow velocity of $0.365 \mathrm{~m} / \mathrm{s}$. This corresponds to $80 \%$ of the critical section averaged flow velocity at a flow depth of $0.15 \mathrm{~m}$. The Reynolds number based on the pier diameter is $\operatorname{Re}=32000$. 


\section{Measurement setup}

The image series of the sediment surface were captured from vertically above in two camera positions by a black and white CMOS-camera at $0.63 \mathrm{Mpx}$ and a frame rate of $27 \mathrm{fps}$. The pixel to grain ratio was found to be $0.145 d_{50}$. A plexiglass plate was slightly submerged on the water surface to provide a defined optical access to the sediment surface. The measurement area was lightened continuously by two spotlights from above and an additional light source inside the cylinder. The setup is sketched in Figure 1.

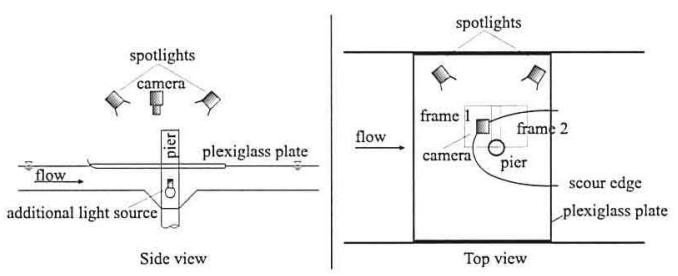

Figure 1: Measurement setup

\section{IMAGE PROCESSING}

The images captured in this setup were analysed by digital image processing techniques. The basic principles are presented in the following section. A detailed description can be found in Pfleger et al. (2010).

\section{Image processing and evaluation algorithm}

Parts of the methods introduced by Papanicolaou et al. (1999) and Radice et al. (2008) were adopted to develop a new algorithm which suits the conditions of the pier scour experiment best.

The image processing is based on the subtraction of the intensity matrices of consecutive frames and the evaluation of non-zero spots in the resulting difference images. To avoid problems with moving uncoloured sediment grains which only have a minor contrast to their background only the movement of black colored grains is detected. In most cases they have a significant contrast to the background except moving above other black grains.

The images are first top-hat filtered to eliminate all non-black pixels and afterwards difference images are produced such that moving black grains appear in the first difference frame in the original position and in the second in their new position. After applying a median filter the non-black pixel groups (the former black grains) are defined to be grains if they meet a certain size criterion. For the grains in the first difference image corresponding grains in the second image are determined via a correlation coefficient. Therefore a maximum movement radius is defined and for grains within this radius the correlation coefficient composed of the gray scale similarity, the deviation from the most probable movement direction in this area (out of a first iteration step) and the exclusion of white grains moving over black background is calculated for each of these grains (Pfleger et al. (2010)). The grain pair with the highest correlation coefficient leads to the calculation of displacement and velocity. 


\section{Perspective calibration}

Due to the three dimensional scour hole surface a perspective calibration of the images is necessary. Therefore geometry measurements of the sediment surface at the investigated stages of the scouring process were made. For each stage an experiment was done for the corresponding time and the geometry was mapped by a laser distance sensor. The topography data are used to conduct a coordinate transformation to determine the real positions that are represented by each pixel.

\section{Postprocessing}

Since only the black grains are detected and the movement intensities in some scour regions are very low, the number of detected displacements in some areas is not high enough to yield sufficient statistics. Thus a spatial median filter is applied to the averaged vector fields. In Pfleger et al. (2010) it is shown that the filter is smoothing the field and not changing the tendencies of the raw pattern.

\section{RESULTS}

The sediment movement patterns of the above described scour experiment were analysed for eight scouring stages (after 5, 30, 60, 120, 240, 480, 720 and $1200 \mathrm{~min}$ ). 40,000 to 50,000 frames per stage were recorded since only the displacements of the black grains were detected and in some areas of the scour hole the movement intensity is very low. Since at most about 10,000 frames (recording time about $6 \mathrm{~min}$ ) can be captured without a considerable change in scour geometry the experiment was run several times to get a satisfying amount of samples.

The temporal evolution of the scour depth shows the typical logarithmic shape. After $1200 \mathrm{~min}$ the deepening rate is lower than one grain diameter per hour and therefore quasi-equilibrium is considered to be reached (Link et al. (2006)).

In Figure 2 the vicinity of the pier is divided in hydrodynamically reasonable sections for a better understanding of the following explanations.

As described in the previous section the measurement system assumes that no suspended transport occurs since all detected movements are projected to the sediment surface. For the transport situation of this experiment the assumption is right for all regions of the scour hole except a small area directly at the backside of the pier (6.1 and the neighboring part of 7 ).

In general all velocities mentioned in the following section are grain velocities.

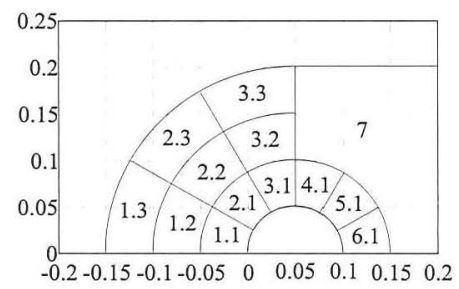

Figure 2: Definition of regions in the pier vicinity 


\section{Mean grain velocity fields}

The mean grain velocities and movement directions were investigated in squared interrogation areas with a length of $0.01 \mathrm{~m}$. In Figure 3 the kinematic patterns of all stages are presented. The background by color represents the ratio of the radial to the tangential component of the grain velocity field with respect to the cylinder axis. Dark gray stands for movement radially inwards, white radially outwards and areas with tangential or no movement are colored as shown in the center of the colormap in Figure 3.

After $5 \mathrm{~min}$ the pattern is dominated by tangential components. Directly at the pier bottom a circular region (1.1-5.1) with high grain velocities can be depicted. Especially in this area almost no radial component appears. Very close to the stagnation plane upstream the cylinder a small area of displacements directed outwards can be seen. This pattern matches with the velocity and bed shear stress fields measured by Hjorth (1975) and Melville (1975) and observations described by Zanke (1982) and Dargahi (1990). The main scouring agent during the initial stage is the accelerated flow at the pier sides. The HV-system is present but weak. The footprint of its rotation can be seen at the pier front but at the pier sides the vortex system is almost eliminated by the tangential flow. This corresponds to the PIV-measurements of Unger and Hager (2007) who showed that the downflow at the pier front is initially mainly deflected in the tangential direction. Outside of this inner ring (everywhere but 1.1-5.1) the grains move mainly in the streamwise direction.

The characteristics of the movements have not changed after $30 \mathrm{~min}$. Upstream the pier the radial components in the inner ring (1.1) become slightly more dominant due to the growth of the HV-system when the scour gets deeper (Melville (1975), Zanke (1982), Dargahi (1990)). Additionally further outside $(1.2,2.2,3.2)$ the grains begin to slide down the scour slope. Thus the component pointing radially inwards grows.

The results after 60 and $120 \mathrm{~min}$ do not differ significantly. The structure of a slowly growing and strengthening $\mathrm{HV}$-system, tangential transport at the pier sides $(2.1,3.1,4.1,5.1)$ and sliding movements along the upper scour slope $(1.2,2.2,3.2)$ are still observable. Especially after $120 \mathrm{~min}$ the magnitude of the tangential vectors at the sides decreases.

For the vector fields after $240,480,720$ and 1200 min a general tendency is noticeable. In the sections 1.2, 2.2, 3.2, 1.3, 2.3 and 3.3 avalanches occur. Below this area $(1.1,2.1,3.1)$ the influence of the HV-system is constantly growing. The contours in Figure 3 show a white area representing the impact of the HV-system on the erosion process. While the scour deepens this ring expands downstream and in radial direction. Near the stagnation plane the ring reaches the pier front, corresponding to the vortex structure e.g. measured by Dey and Raikar (2007) or Unger and Hager (2007). Following the pier side downstream after 240 and $480 \mathrm{~min}$ an area of tangential movement is present where the influence of the tangentially deflected downflow is evident $(2.1,3.1,4.1,5.1)$. This tangential movement decreases during the further process. The effective range of the HV-system expands towards the pier in the upstream half of the scour $(1.1,2.1,3.1)$. This corresponds to the vortex size evolution measurements by Muzzammil and Gangadhariah (2003). The influence of the accelerated tangential flow components decreases. 

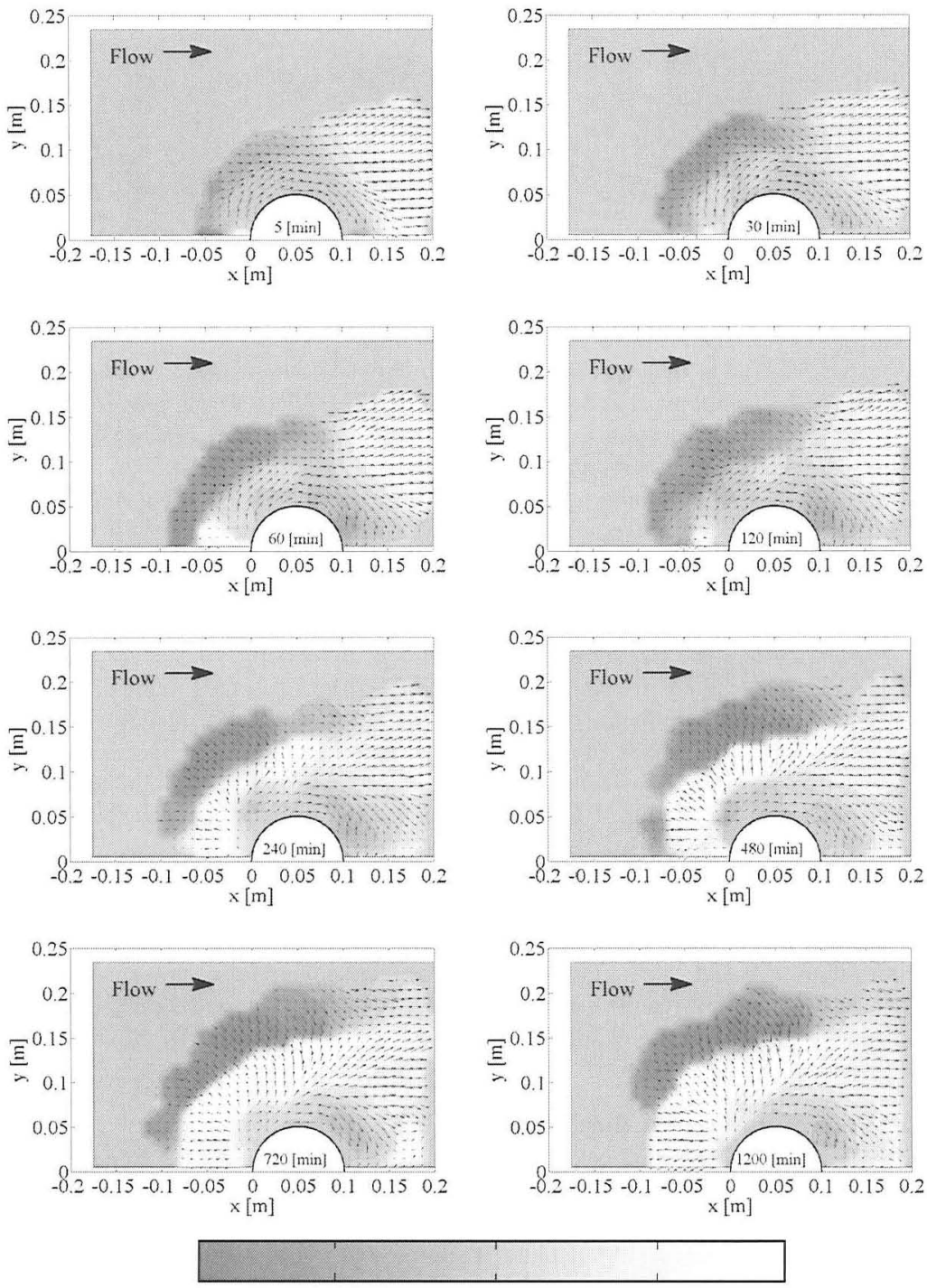

radial inwards

tangential

radial outwards

Figure 3: Mean grain velocity vector field with contour of the ratio of radial to tangential components 

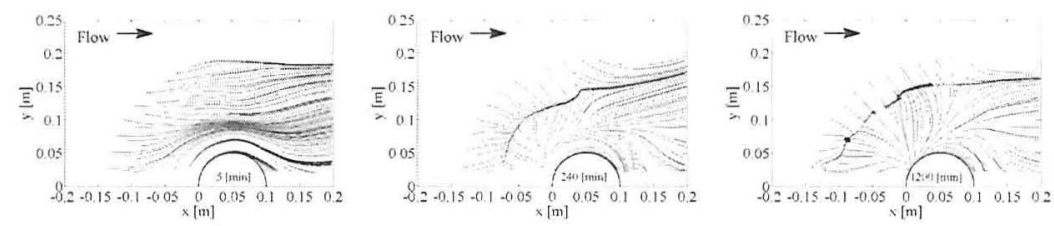

Figure 4: Streamlines of sediment movement after 5, 240 and $1200 \mathrm{~min}$

In the downstream half of the scour hole initially sediment is deposited and forms a small hill (7). At the outer side of this hill streamwise movement is superposed by a slight sliding component directed down the hillside. Close to the pier $(5.1,6.1)$ for all scouring stages the erosive effect of a small detachment zone (non uniform movement directions) can be observed.

In Figure 4 the streamline plots of the sediment movement after 5,240 and $1200 \mathrm{~min}$ are shown. The patterns agree very well with the mean near-bed velocity directions shown in Melville (1975) for the initial, the intermediate and the equilibrium scour hole respectively. The change of the main erosion directions in between these three stages is evident. This reveals the processes described before.

Figure 5 shows the magnitude of the mean grain velocity vectors after 5,240 and $1200 \mathrm{~min}$. The values are normalized by the section averaged velocity of the channel flow $u_{-} b u l k$. After $5 \mathrm{~min}$ the highest values appear at the upstream facing cylinder sides $(2.1,3.1)$ where the scouring process starts and downstream of the cylinder where the grains are transported off the scour hole (7). The mean grain velocities are lower than $30 \%$ of the approaching flow velocity even in the initial stage. The grain velocities in the later stages are in general lower than at the beginning. This is especially essential in the upstream half where the transport is now dominated by the HV-system and avalanches in the upper slope. In this part a ring with very low mean grain velocities appears approximately in the middle of the scour slope $(1.2,2.2,3.2)$. This is where the two phenomena converge and an unstable rim is formed (Melville and Raudkivi (1977)). During the scouring process the velocities downstream the cylinder where the grains are transported off the scour hole (7) decrease significantly.

\section{Scatterplots of movement directions}

Since the averaged grain velocity values do not fully represent the dynamics of the erosion process scatterplots of the two cartesian velocity components are shown for every second interrogation area in Figure 6. Each point corresponds to one measured combination of grain velocity components in the $\mathrm{x}$ - and $\mathrm{y}$-direction within this area. The scour hole is shown after 5 and $240 \mathrm{~min}$. As described for the mean value plots the properties of the intermediate and the equilibrium scour hole are very similar.

In the initial stage the point clouds in the high velocity area at the upstream facing pier sides $(1.1,2.1,3.1)$ are relatively compact. In the section $1.2,2.2$ and 3.2 the data points are distributed across a wider area in many directions. This could be referred to an already established small detachment zone along the scour edge. 
Close to the stagnation plane the influence of the growing HV-system scatters the points significantly. In the detachment zone in the wake of the pier $(5.1,6.1)$ the grain movements fill all quadrants of the scatterplots equally. In section 7 where the movement direction is comparatively straight out of the scour the allocation of the displacement events is uniform.

After 240 min the influence of the HV-system can be distinguished (1.1-3.1, 1.2-3.2). Even if there are quite distinct mean movement directions in this area the displacements are spread over a wide range. This refers to the uphill transport by the HV and the temporary collapsing rim. Additionally the highly turbulent nature of the HV-system leads to spatially and temporarily fluctuating transport events. In the downstream half of the scour the data points are again less scattered (7). Here the less fluctuating accelerated flow components dominate the erosion process.

\section{Transport intensities}

In Figure 7 the transport intensities are represented by the number of displaced grains per second and square centimetre.

After $5 \mathrm{~min}$ in the upstream part a concentrically shaped area with high transport intensities is evident (2.1-4.1). Here also relatively high grain velocity magnitudes appear which lead to an effective transport. Inside this ring close to the pier the number of moving grains is significantly lower. The grain velocities are comparable but the tangentially accelerated flow as the main scouring agent reaches the scour bottom not constantly. An explanation for this is that the already weakly acting HV-system pushes the effective flow components temporarily away from the pier sides. In the downstream part high but not effective transport intensities in the detachment zone $(5.1,6.1)$ and considerably smaller but uniform transport events in section 7 can be observed.

For the intermediate and the equilibrium scour hole the HV-system causes high intensities in its effective range (1.2-3.2). In contrast to the equilibrium stage after $240 \mathrm{~min}$ many grains are also displaced in the sections 5.1 and 6.1 pointing to a detached flow. This matches with the velocity measurement in horizontal planes by Unger and Hager (2007) who state that inside a developed scour hole the flow does not detach from the cylinder. In general the transport intensities after $1200 \mathrm{~min}$ tend to zero.
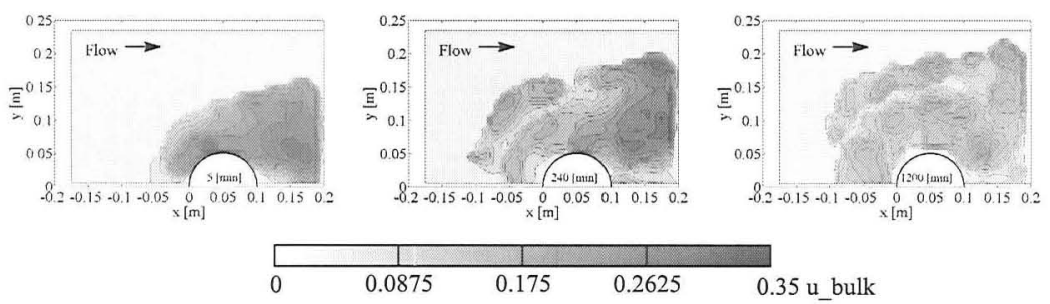

Figure 5: Velocity magnitudes of sediment movement after 5, 240 and $1200 \mathrm{~min}$ 

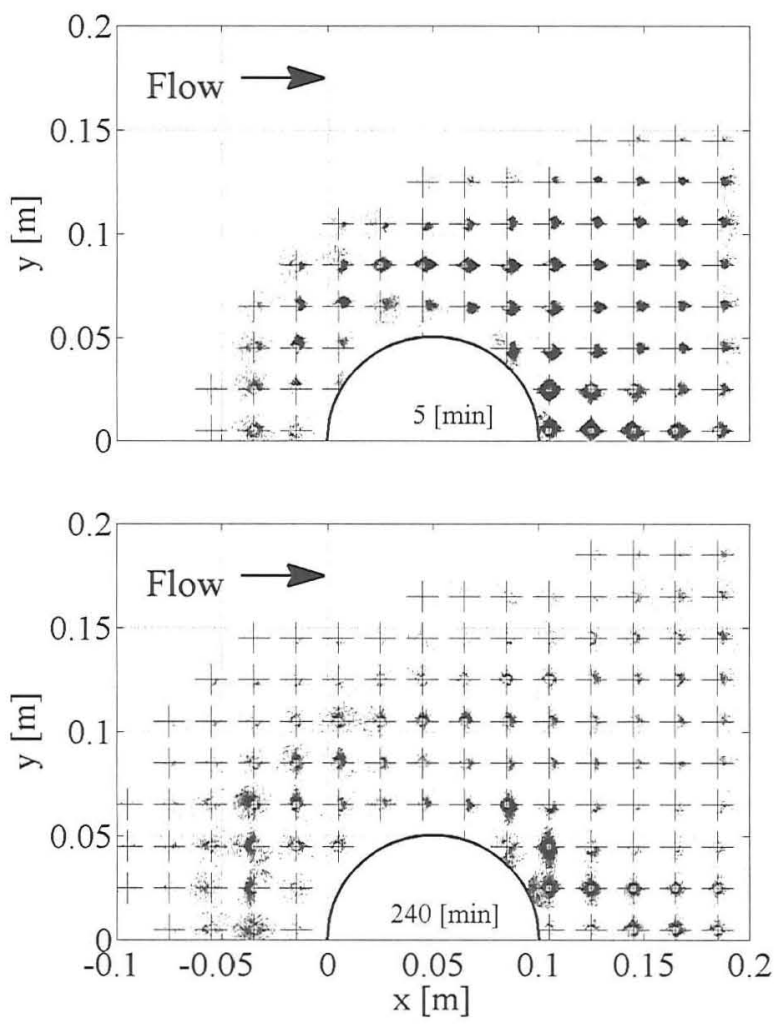

Figure 6: Scatterplots of instantaneous movement events after 5 and $240 \mathrm{~min}$

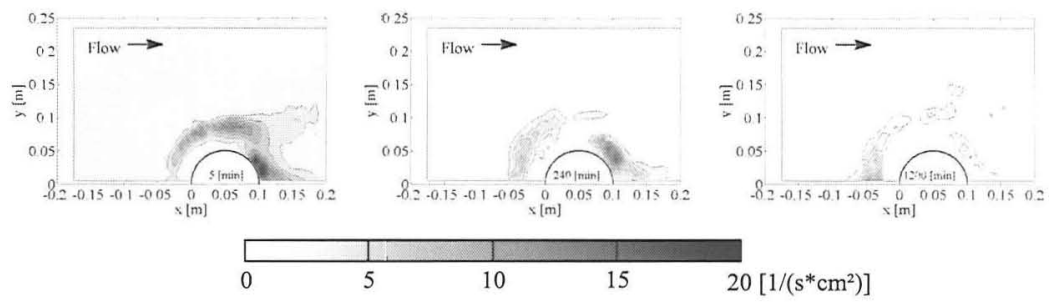

Figure 7: Number of displaced grains $\left[1 /\left(\mathrm{s} \cdot \mathrm{cm}^{2}\right)\right]$ after 5, 240 and $1200 \mathrm{~min}$ 


\section{CONCLUSION AND OUTLOOK}

The initial scour hole formation is mainly caused by tangentially accelerated flow components. This results in relatively uniform movement directions at the pier sides and in the downstream part at comparably high mean grain velocities. The combination of mainly downstream directed grain movements and high erosion rates leads to a rapid scour growth. In the growing scour the influence of the $\mathrm{HV}$-system on the erosion is increased. The caused movements are a combination of uphill transport by the HV and avalanches due to temporarily collapses of the scour slope. Thereby a rim is established in the middle of the slope. The contribution of these displacements to the mass transport off the scour is small since the two described transport effects have almost opposite directions. In the regions where the effective transport out of the scour takes place the intensities and grain velocity magnitudes are decreasing. This combination leads to the deceleration of the scouring process.

In a continuative work near wall LDA measurements in the scour region will be conducted. In a fixed scour hole geometry mean velocities and turbulence intensities will be measured and combined with the results of this study to gain more detailed dependencies between flow field and sediment transport within a scour hole.

\section{REFERENCES}

Dargahi, B. (1990). "Controlling mechanism of local scouring:" Journal of Hydraulic Engineering, 116(10), 1197-1214.

Dey, S. and Raikar, R. (2007). "Characteristics of horseshoe vortex in developing scour holes at piers." Journal of Hydraulic Engineering, 133(4), 399-413.

Hjorth, P. (1975). "Studies on the nature of local scour," PhD thesis, University of Lund.

Keshavarzy, A. and Ball, J. (1999). "An application of image processing in the study of sediment motion." Journal of Hydraulic Research, 37(4), 559-576.

Link, O. (2006). "Untersuchung der Kolkung an einem schlanken zylindrischen Pfeiler in sandigem Boden," PhD thesis, Technische Universität Darmstadt.

Link, O., Pfleger, F., and Zanke, U. (2006). "Automatic non-intrusive measurement of scour-hole geometry." Proceedings to ICSE3, H. Verheij and G. Hoffmans, eds., 3rd International Conference on Scour and Erosion, Gouda, The Netherlands. CURNET, 403-408.

Melville, B. W. (1975). "Local scour at bridge sites," PhD thesis, University of Auckland.

Melville, B. W. and Raudkivi, A. (1977). "Flow characteristics in local scour at bridge piers." Journal of Hydraulic Research, 15(4), 373-380.

Muzzammil, M. and Gangadhariah, T. (2003). "The mean characteristics of horseshoe vortex at a cylindrical pier." Journal of Hydraulic Research, 41(3), 285-297.

Papanicolaou, A. N., Diplas, P., Balakrishnan, M., and Dancey, C. L. (1999). "Computer vision technique for tracking bed load movement." Journal of Computation in Civil Engineering, 71-79.

Pfleger, F., Rapp, C., and Manhart, M. (2010). "Experimental investigation on the sediment movement in the vicinity of a cylindrical bridge pier." Proceedings to Riverflow conference 2010. Accepted for publication.

Pilotti, M., Menduni, G., and Castelli, E. (1997). "Monitoring the inception of sediment transport by image processing techniques." Experiments in Fluids, 23, 202-208.

Radice, A., Malavasi, S., and Ballio, F. (2008). "Sediment kinematics in abutment scour." Journal of Hydraulic Engineering, 134(2), 146-156.

Sechet, P. and LeGuennec, B. (1999). "Bursting phenomenon and incipient motion of solid particles in bed-load transport." Journal of Hydraulic Research, 37(5), 683-696.

Unger, J. and Hager, W. (2007). "Down-flow and horseshoe vortex characteristics of sediment embedded bridge piers." Exp Fluids, 42, 1-19.

Zanke, U. (1982). "Kolke am Pfeiler in richtungskonstanter Strömung und unter Welleneinfluss." Mitteilungen des Franzius-Instituts für Wasserbau- und Küsteningenieurwesen der Universität Hannover, 54, 381-416. 